\title{
Relation between weather radar equation and first-order backscattering theory
}

\author{
F. S. Marzano and G. Ferrauto \\ Centro di Eccellenza CETEMPS, Dipartimento di Ingegneria Elettrica, Università dell'Aquila, Monteluco di Roio, 67040 \\ L'Aquila, Italy
}

Received: 29 October 2002 - Published in Atmos. Chem. Phys. Discuss.: 13 January 2003

Revised: 14 May 2003 - Accepted: 4 June 2003 - Published: 20 June 2003

\begin{abstract}
Aim of this work is to provide a new insight into the physical basis of the meteorological-radar theory in attenuating media. Starting form the general integral form of the weather radar equation, a modified form of the classical weather radar equation at attenuating wavelength is derived. This modified radar equation includes a new parameter, called the range-bin extinction factor, taking into account the rainfall path attenuation within each range bin. It is shown that, only in the case of low-to-moderate attenuating media, the classical radar equation at attenuating wavelength can be used. These theoretical results are corroborated by using the radiative transfer theory where multiple scattering phenomena can be quantified. From a new definition of the radar reflectivity, in terms of backscattered specific intensity, a generalised radar equation is deduced. Within the assumption of first-order backscattering, the generalised radar equation is reduced to the modified radar equation, previously obtained. This analysis supports the conclusion that the description of radar observations at attenuating wavelength should include, in principle, first-order scattering effects. Numerical simulations are performed by using statistical relationships among the radar reflectivity, rain rate and specific attenuation, derived from literature. Results confirm that the effect of the range-bin extinction factor, depending on the considered frequency and range resolution, can be significant at $\mathrm{X}$ band for intense rain, while at Ka band and above it can become appreciable even for moderate rain. A discussion on the impact of these theoretical and numerical results is finally included.
\end{abstract}

Correspondence to: F. S. Marzano or G. Ferrauto (marzano@ing.univaq.it, ferrauto@ing.univaq.it)

\section{Introduction}

A well established technique to retrieve rainfall structure and microphysics (Atlas et al., 1990) is based on the use of weather radars. Optimal frequency bands for rain radar meteorology are generally chosen, on one hand, to enhance the sensitivity of microwave backscattering to hydrometeor volumetric distribution and, on the other hand, to reduce the effects of path attenuation (Bringi and Chandrasekar, 2001). In this respect, $\mathrm{S}$ band is probably one of the best choices and is typically used for ground-based installations. However, due to constraints on the component sizes and high power requested at $\mathrm{S}$ band, higher radar frequencies have been also considered for operational purposes (Sauvageot, 1992). For ground-based radar systems, C-band frequency has been widely adopted for the operational case, while for space-based sensors frequency bands from $\mathrm{Ku}$ to $\mathrm{W}$ have been taken into consideration for cloud and rain retrieval (e.g., Delrieu et al., 2000; Meneghini et al., 1983).

When operating a radar at attenuating wavelength, path attenuation needs to be included in the equation governing the quantitative analysis of backscattering measurements (Meneghini, 1978; Sauvageot, 1992). The classical radar equation in an attenuating medium, as generally stated, takes into account the single scattering due to raindrops, weighted by the two-way path attenuation from the considered range gate to the radar antenna. As known, hydrometeor path attenuation increases as the frequency increases beyond $\mathrm{S}$ band (Sauvageot, 1992). Any radar technique above $\mathrm{S}$ band should take into account, and possibly remove, path attenuation effects in order to correctly convert measured reflectivity into rain rate. To this aim, iterative and constrained methods have been proposed to process radar data both from ground-based and from space-based systems (e.g., Meneghini, 1978; Aydin et al., 1989; Marzano et al., 1999; Serrar et al., 2000; Testud et al., 2000). 
Nevertheless, the validity of the classical radar equation in attenuating media is not clearly asserted in literature. The theoretical framework behind the classical radar equation generally resorts to the Friis equation considering, in the farfield zone, a locally uniform plane-wave propagation attenuated along the path following an inverse-exponential law (e.g., Bogush, 1989). For frequencies higher than S band and depending on the rainfall intensity, the increase of path attenuation is also related to the increase of the volumetric albedo and scattering asymmetry of hydrometeors (Ishimaru, 1978; Marzano et al., 2000; de Wolf et al., 2000). This means that the contribution of first and successive orders of scattering can be appreciable in determining the radar received power. A careful analysis of the validity of the classical radar equation could be obtained only if the radar equation itself is framed within a more general theory which can include the effects of incoherent scattering phenomena (Marzano et al., 2003).

In this work, a new insight into the physical basis of radar observation theory in attenuating media is proposed. Starting form the general integral form of the weather radar equation, a modified form of the classical weather radar equation at attenuating wavelength is derived. This modified radar equation includes a new parameter, called the range-bin extinction factor, taking into account the rainfall path attenuation within each range bin. These theoretical results are also obtained by using a microwave radiative transfer approach, described by means of an integro-differential equation. It is demonstrated that, in strong attenuating media, the radar equation should include first-order scattering effects. In the last section numerical simulations of the range-bin extinction factor are presented by using statistical relations between reflectivity, rain rate and specific attenuation, derived from literature, together with a discussion of the possible impact of the obtained results. Practical applications, such effects on radar inversion algorithms and spatial average of measured reflectivities at attenuating wavelengths, are eventually addressed.

\section{Modified radar equation at attenuating wavelength}

In the present and following section, we refer to the classical radar equation at attenuating wavelength as an equation having the following expression (e.g., Bogush, 1989; Sauvageot, 1992):

$<P_{R}\left(r, \Omega_{0}\right)>=C \frac{Z_{e}\left(r, \Omega_{0}\right)}{r^{2}} L^{2}(r)=C \frac{Z_{m}\left(r, \Omega_{0}\right)}{r^{2}}$,

where $<P_{R}\left(r, \Omega_{0}\right)>$ is the mean received power, obtained from averaging radar echoes due to the scattering volume $\Delta V_{r}$ at a range $r$ in the pointing direction indicated by the solid angle $\Omega_{0}, C$ is the instrumental constant. In (1) $Z_{e}$ is the equivalent reflectivity factor, $L$ is the one-way path attenuation at range $r$ and $Z_{m}$ is the measured reflectivity factor such that, in this case, $Z_{m}=Z_{e} L^{2}$.
In this paper the classical radar equation in attenuating media, given by (1), is derived by using two different approaches. On one hand, the integral radar equation is here integrated to obtain first the modified radar equation and, then, the classical radar equation. On the other hand, the same modified radar equation is obtained using the first-order scattering solution within the radiative transfer theory by using a generalised definition of the radar reflectivity.

The integral equation of a pulsed weather radar, operating in an attenuating medium, basically relates the mean received power $<P_{R}\left(r, \Omega_{0}\right)>$ to the transmitted peak power $P_{T}$. Let us suppose an observation geometry, where the radar is placed in the origin. For simplicity of notation, besides the spherical coordinates $(r, \theta, \phi)$ with respect to the $z$-axis, we introduce a slant reference coordinate system $\left(r^{\prime}, \theta_{r}, \phi_{r}\right)$ with the corresponding solid angle $\Omega_{r}=\left(\theta_{r}, \phi_{r}\right)$. The latter is such that its vertical coordinate $z_{r}$ coincides with the radar range $r$ along the pointing angle $\Omega_{0}=\left(\theta_{0}, \phi_{0}\right)$ so that $\theta_{r}$ stands for the angle between $r$ and $r^{\prime}$. Of course, when $\theta_{0}=0$ and $\phi_{0}=0, \Omega_{0}=(0,0)$ indicates a zenith (or nadir) observation along $z$ and the coordinates $(r, \theta, \phi)$ coincide with $\left(r^{\prime}, \theta_{r}, \phi_{r}\right)$.

In the presence of an inhomogeneous attenuating medium, the integral radar equation can be stated as follows (Meneghini et al., 1983; Savaugeot, 1992):

$<P_{R}\left(r, \Omega_{0}\right)>=\frac{P_{T} \lambda^{2}}{(4 \pi)^{3}} \int_{\Delta V_{r}} \eta\left(r^{\prime}, \Omega_{r}\right) \frac{G^{2}\left(\Omega_{r}\right)}{r^{\prime 2}} e^{-2 \tau_{r}\left(r^{\prime}\right)} d^{3} r^{\prime}$,

where $\lambda$ is the radar wavelength (in vacuum), $\Delta V_{r}$ is the radar resolution volume or cell (spanned by coordinates $r^{\prime}$, $\theta_{r}$ and $\left.\phi_{r}\right), \eta$ is the volumetric radar reflectivity, $\tau_{r}$ is the optical thickness (or path attenuation $A$ ) along the range $r$, $d^{3} r^{\prime}$ is the elementary volume within the radar bin. The antenna gain function $G\left(\Omega_{r}\right)$ along $\Omega_{r}$ is such that $G\left(\Omega_{r}\right)=$ $G_{0}\left|f_{n}\left(\Omega_{r}\right)\right|^{2}$, with $G_{0}=G\left(\Omega_{0}\right)$ the maximum gain in the pointing direction $\Omega_{0}$ and $\left|f_{n}\left(\Omega_{r}\right)\right|^{2}$ the one-way normalized radiation pattern. The volumetric radar reflectivity $\eta$ in (2) can be related to the equivalent reflectivity factor $Z_{e}$ by the well-known relation (Sauvageot, 1992):

$\eta\left(r, \Omega_{r}\right) \equiv \frac{\pi^{5}|K|^{2}}{\lambda^{4}} Z_{e}\left(r, \Omega_{r}\right)$,

where $K$ is the medium polarizability complex factor.

It is worth mentioning that a path attenuation factor appears in (2) as a term of the integral expression and includes both the extinction within the considered range bin and from the range bin to the radar antenna (Meneghini et al., 1983). The one-way path attenuation factor from the radar antenna to the considered range bin $L$ can be expressed as:

$L\left(r, \Omega_{r}\right) \cong L\left(r, \Omega_{0}\right) \equiv L(r)=e^{-\tau_{r}(r)}=e^{-\int_{0}^{r} k\left(r^{\prime}\right) d r^{\prime}}$,

being $k$ the volumetric specific attenuation (or extinction coefficient). Note that we prefer here to indicate the path attenuation with $\tau_{r}$ to be consistent with the radiative transfer 
notation, used in the next section, even though $\tau$ generally stands for the radar pulse length in radar theory. Equation (4) is derived under the assumption of a weak dependence of $L$ on the observation angle $\Omega_{r}$ within the radar beam.

The validity of (2) is, of course, limited to scalar radiation (e.g., single polarization radar), even though the theoretical arguments discussed below can be modified by considering a polarimetric form of (2) (e.g., Bringi and Chandrasekar, 2001).

If the integration in (2) is modified to the finite range bin $\Delta r$ (i.e., range resolution) and to the antenna main-lobe beamwidth $\Omega_{M}$ (defined as either the $-3 \mathrm{~dB}$ or the $-6 \mathrm{~dB}$ solid angle), (2) can be readily expressed in a simplified form. Moreover, if the radar antenna is sufficiently directive such that the scattering volume $\Delta V_{r}$ can be assumed to be uniformly filled by randomly-distributed scatterers, the equivalent reflectivity factor $Z_{e}$ and specific attenuation $k$ become independent on the angle $\Omega_{r}$ and range $r^{\prime}$ within the radar volume. In these circumstances, (2) can be simplified as follows:

$$
\begin{aligned}
& <P_{R}\left(r, \Omega_{0}\right)>\cong \frac{P_{T} \lambda^{2}}{(4 \pi)^{3}} \frac{\pi^{5}|K|^{2}}{\lambda^{4}} \\
& G_{0}^{2} Z_{e}\left(r, \Omega_{0}\right) \Omega_{2 A} \int_{r}^{r+\Delta r} \frac{e^{-2 k r^{\prime}}}{r^{\prime 2}} d r^{\prime},
\end{aligned}
$$

where $\Omega_{2 A}$ is the antenna two-way radiation solid angle, defined by:

$\Omega_{2 A} \equiv \int_{\Omega_{M}}\left|f_{n}\left(\Omega_{r}\right)\right|^{4} d \Omega_{r}$.

If the antenna power pattern is assumed to be Gaussian, the expression of $\Omega_{2 A}$ in (6) yields the well-known Probert-Jones correction factor to the radar equation (Sauvageot, 1992).

The last integral in (5) can be easily calculated. Assuming $\Delta_{r}<<r$, that is for ranges much larger than the range resolution, the mean received power from an arbitrary range bin can be, thus, re-arranged as follows:

$$
\begin{aligned}
& <P_{R}\left(r, \Omega_{0}\right)>\cong \\
& \frac{P_{T} \pi^{2}|K|^{2} G_{0}^{2} \Omega_{2 A} \Delta_{r}}{64 \lambda^{2}} \frac{Z_{e}\left(r, \Omega_{0}\right) f_{b}(\Delta r)}{r^{2}} L^{2}(r),
\end{aligned}
$$

where $f_{b}(r)$ is the range-bin extinction factor, defined by:

$f_{b}(\Delta r) \equiv \frac{1}{2 k \Delta r}\left(1-e^{-2 k \Delta r}\right)=\frac{1}{2 \Delta \tau_{r}(r)}\left(1-e^{-2 \Delta \tau_{r}(r)}\right)$

with $\Delta \tau_{r}$ the range-bin optical thickness. Equation (8) expresses the effect of specific attenuation and/or range resolution within the considered radar scattering volume.

A comparison of (7) with the classical radar equation in (1) suggests a bin-averaged equivalent reflectivity factor $Z_{e b}\left(r, \Omega_{0}\right)$ at a range $\mathrm{r}$ can be defined as:

$Z_{e b}\left(r, \Omega_{0}\right) \equiv Z_{e}\left(r, \Omega_{0}\right) f_{b}(\Delta r)$. so that the mean received power can be put into the following modified radar equation:

$<P_{R}\left(r, \Omega_{0}\right)>\cong C \frac{Z_{e b}\left(r, \Omega_{0}\right)}{r^{2}} L^{2}(r)$.

The radar instrumental constant $C$ in (10) is given by:

$C \equiv \frac{P_{T} \pi^{2}|K|^{2} G_{0}^{2} \Omega_{2 A} \Delta r}{64 \lambda^{2}}$,

being $\Delta r=c \Delta t / 2$ the radar range resolution, with $\Delta t$ the pulse width and $c$ the speed of light (in vacuum). When the output signal is characterised at the receiver output port, the instrumental constant $C$ can include receiver processing effects as well, such as the finite bandwidth power loss, matched filter weighting and receiver insertion losses (e.g., Sauvageot, 1992). The receiver noise power is here not considered being not essential to explain the core of our thesis.

It is worth mentioning that, if either the specific attenuation $k$ is small or the range resolution $\Delta r$ is small so that the range-bin optical thickness $\Delta \tau_{r}$ is much less than 1 , then the range-bin extinction factor in (8) reduces to:

$f_{b}(\Delta r) \cong \frac{1}{2 \Delta \tau_{r}(r)}\left[1-\left(1-2 \Delta \tau_{r}(r)\right)\right] \cong 1$.

Under these conditions, the modified radar equation becomes identical to the classical radar equation in attenuating media, given by (1). We can conclude that the classical radar equation is a particular case of the modified radar Eq. (10) when extinction effects within each range bin are not significant.

\section{Weather radar equation within radiative transfer theory}

Scattering and propagation characteristics of electromagnetic waves through a random medium can be profitably studied by using the radiative transfer theory (Tsang et al., 1985). The radiative transfer theory (RTT) is diffusely applied in passive and active remote sensing, even though not generally considered in classical radar meteorology approaches (Atlas et al., 1990). One of the reasons is probably due to the negligibility of multiple scattering phenomena in most applications of weather radars at frequencies below $10 \mathrm{GHz}$.

The need to consider higher operating frequencies has recently raised the issue of quantifying incoherent backscattered radiation in radar meteorology problems (Marzano et al., 2000; de Wolf et al., 2000; Marzano and Ferrauto, 2002). In this section our goal is to demonstrate that the modified radar equation, deduced from the integral radar equation and slightly extending the classical radar equation, has the same form of the first-order backscattering solution of the radiative transfer equation.

The fundamental quantity in the radiative transfer theory is the specific intensity $I$, also called radiance (Ishimaru, 1978). The specific intensity of a radiation of frequency $v$ 
is defined as the average power flux density within a unit frequency band, centred at $v$, and within a unit solid angle. The specific intensity $I$ is, consequently, measured in $\left[\mathrm{W} \mathrm{m}^{-2}\right.$ $\mathrm{sr}^{-1} \mathrm{~Hz}^{-1}$ ]. Under the assumption of unpolarized radiation, the specific intensity of the scattered radiation is the solution of a scalar differential-integral equation, known as the radiative transfer equation (Ishimaru, 1978; Tsang et al., 1985; Marzano et al., 1999).

Before dealing with the solution of the radiative transfer equation (RTE), it is opportune to deduce a generalised version of the radar equation expressed in terms of the received specific intensity, possibly due to multiple scattering. In order to do this, the backscattered specific intensity, incident on the radar antenna, can be expressed by means of the apparent radar reflectivity of the considered range bin (Marzano et al., 2000; Marzano et al., 2003).

\subsection{Generalised radar equation at attenuating wavelength}

If $\Delta r$ is the radial resolution and $P_{T}$ is the transmitted power along the direction $\Omega_{0}=\left(\theta_{0}, \phi_{0}\right)$, then the transmitted power flux density at range $r$ in a given direction, opposite to the receiving one $\Omega_{r}$, can be related to $P_{T}$ through the antenna gain definition:

$F_{T}(r)=\frac{P_{T} G\left(\Omega_{r}\right)}{4 \pi r^{2}}$

where, again, $G\left(\Omega_{r}\right)$ is the antenna gain function. The transmitted power $P_{T}$ is partially reflected backwards by each particle intercepting the radar beam within the scattering volume.

The apparent radar reflectivity $\eta_{a}$, defined as the ensemble average of backscattering cross sections of all particles within a unit volume, can be related to the average value of the backscattered specific intensity $I_{R}\left(r, \Omega_{r}\right)$ by means of the following relationship (Marzano et al., 2000, 2002):

$\eta_{a}\left(r, \Omega_{r}\right)=\frac{4 \pi}{\Delta r} \frac{<I_{R}\left(r, \Omega_{r}\right)>}{F_{T}(r)}$.

In analogy to (3), the apparent reflectivity $\eta_{a}$ can be expressed through the apparent equivalent reflectivity factor $Z_{a}$ :

$Z_{a}\left(r, \Omega_{r}\right) \equiv \frac{\lambda^{4}}{\pi^{5}|K|^{2}} \eta_{a}\left(r, \Omega_{r}\right)=$

$\frac{\lambda^{4}}{\pi^{5}|K|^{2}}\left[\frac{4 \pi}{\Delta r} \frac{<I_{R}\left(r, \Omega_{r}\right)>}{F_{T}(r)}\right]$.

Given the mean value $<I_{R}\left(r, \Omega_{r}\right)>$ of the apparent received specific intensity, the apparent backscattered received power $<P_{R a}\left(r, \Omega_{0}\right)>$ can be expressed as (Ishimaru, 1978; Tsang et al., 1985):

$<P_{R a}\left(r, \Omega_{0}\right)>=\frac{\lambda^{2}}{4 \pi} \int_{4 \pi} G\left(\Omega_{r}\right)<I_{R}\left(r, \Omega_{r}\right)>d \Omega_{r}$, where the directive gain $G$ has been related to the antenna equivalent area $A_{e}$ through the reciprocity formula $G_{0}=$ $\left(4 \pi / \lambda^{2}\right) A_{e 0}$.

Thus, substituting (13) and (14) into (16), we have:

$<P_{R a}\left(r, \Omega_{0}\right)>=$

$\frac{\lambda^{2} \Delta r}{(4 \pi)^{2}} \int_{4 \pi} G\left(\Omega_{r}\right) \eta_{a}\left(r, \Omega_{r}\right) \frac{G\left(\Omega_{r}\right)}{4 \pi} \frac{P_{T}}{r^{2}} d \Omega_{r}$.

If $<I_{R}\left(r, \Omega_{r}\right)>$, and thus $\eta_{a}\left(r, \Omega_{r}\right)$, can be assumed constant within the radar scattering volume, it is straightforward to re-express the mean apparent received power. Finally, from (17) we can obtain a generalised radar equation:

$<P_{R a}\left(r, \Omega_{0}\right)>\cong C \frac{Z_{a}\left(r, \Omega_{0}\right)}{r^{2}}$,

where the radar constant $C$ is given by:

$C=\frac{G_{0}^{2} \Omega_{2 A} \Delta r \pi^{2}|K|^{2}}{64 \lambda^{2}}$

Equation (18) is formally identical to the classical radar equation in (1). In particular, this equivalence is readily obtained when the measured reflectivity factor $Z_{m}\left(r, \Omega_{0}\right)$ in (1) is expressed by means of the apparent reflectivity factor $Z_{a}\left(r, \Omega_{0}\right)$. However, the generalised radar equation (18) has a different physical meaning and a more general validity with respect to the classical and modified radar equations since:

1. it can take into account multiple scattering effects to any order of scattering, being the apparent radar reflectivity related to specific intensity solution of the radiative transfer equation (Marzano et al., 2000, 2003);

2. it includes, as a particular case, the modified radar equation if the apparent reflectivity factor is expressed in terms of the equivalent reflectivity factor $Z_{e}$ and the binaveraged extinction factor $f_{b}$, i.e.:

$$
\begin{aligned}
& Z_{a}\left(r, \Omega_{0}\right) \equiv Z_{e b}\left(r, \Omega_{0}\right) L^{2}(r)= \\
& Z_{e}\left(r, \Omega_{0}\right) f_{b}(\Delta r) L^{2}(r) .
\end{aligned}
$$

3. it includes, as a particular case, the classical radar equation if $Z_{a}\left(r, \Omega_{0}\right)=Z_{e}\left(r, \Omega_{0}\right) L^{2}(r)$ in (18).

3.2 Apparent equivalent reflectivity and first-order backscattering

The aim of this section is to derived an explicit expression of $Z_{a}$, given in (15), from the radiative transfer equation. In a way, we would like to answer to the following questions:

- under what assumptions the RTT solution can give an expression of $Z_{a}$ which reduces the generalised radar equation to the modified one? 
- to this scope, can we limit our analysis to the firstorder backscattering theory following an iterative solution method?

Let us consider a simple atmospheric model consisting of a single homogeneous atmospheric slab whose bounds are, in terms of optical thickness, $\tau=0$ and $\tau=\Delta \tau_{r}$. If the specific attenuation $k$ is constant, then the geometrical thickness of the slab is $\Delta r=\tau_{r} / k$. The radar antenna is placed in $\tau=0$. Note that the analysis of a single homogeneous slab of optical thickness $\Delta \tau_{r}$ is equivalent to consider, in a classical radar context, a range bin of resolution $\Delta r$ with $L(r)=0$.

Under the above mentioned assumptions and by applying proper boundary conditions, the diffuse specific intensity in the first-order scattering (FOS) approximation for backward directions is given by (Ishimaru, 1978; Tsang et al., 1985) :

$$
\begin{aligned}
& I^{(1)}\left(0, \Omega_{0}\right)=<I_{R}\left(r, \Omega_{0}\right)>_{F O S}= \\
& \frac{w F_{0}}{8 \pi} p\left(\Omega_{0},-\Omega_{0}\right)\left(1-e^{-2 \Delta \tau_{r}}\right),
\end{aligned}
$$

where $p\left(\Omega_{0},-\Omega\right)$ is the phase function in the backward direction, $w=k_{s} / k$ is the single scattering albedo with $k_{s}$ the volumetric scattering coefficient and $F_{T}(r)=F_{0}$. Notice that the FOS backward radiance $I^{(1)}$ has been interpreted as the mean received specific intensity $\left\langle I_{R}\left(r, \Omega_{0}\right)>\right.$, due to the ensemble averaging of backscattered radiation from the polydispersion of particles within the rain slab when firstorder scattering is the dominant contribution (Marzano et al., 2003).

The above expression, substituted in (14), allows one to calculate the apparent radar reflectivity, due to a resolution volume of slant depth $\Delta r$, within the FOS approximation:

$$
\begin{aligned}
& \eta_{a F O S}\left(r, \Omega_{0}\right)=\frac{4 \pi}{\Delta r} \frac{<I_{R}\left(r, \Omega_{0}\right)>_{F O S}}{F_{0}}= \\
& \frac{k_{s} p\left(\Omega_{0},-\Omega_{0}\right)}{2 k \Delta r}\left(1-e^{-2 \Delta \tau_{r}}\right) .
\end{aligned}
$$

This equation can also be written as:

$\eta_{a F O S}\left(r, \Omega_{0}\right)=\eta\left(r, \Omega_{0}\right) \frac{1}{2 \Delta \tau_{r}}\left(1-e^{-2 \Delta \tau_{r}}\right)$,

being $\Delta \tau_{r}=k \Delta r$ for the slab geometry considered. By definition, the radar reflectivity $\eta$ in (23) is given by (Ishimaru, 1978; Marzano et al., 2000):

$\eta\left(r, \Omega_{0}\right)=p\left(\Omega_{0},-\Omega_{0}\right) k_{s}$.

Converting (24) into the analogous expression in terms of the equivalent reflectivity factor using (3), we obtain the fundamental result:

$Z_{a F O S}\left(r, \Omega_{0}\right)=Z_{e}\left(r, \Omega_{0}\right) \frac{\left(1-e^{-2 \Delta \tau_{r}}\right)}{2 \Delta \tau_{r}}$.

In the second member of (25) we recognize the range-bin extinction factor $f_{b}(r)$, defined in (8). Thus, an identical expression has been found for both the FOS apparent equivalent reflectivity factor $Z_{a}$ FOS and the bin-averaged equivalent reflectivity factor $Z_{e b}$, given in (9) when $L=0$, that is:

$$
\begin{aligned}
& Z_{a F O S}\left(r, \Omega_{0}\right) \equiv Z_{e}\left(r, \Omega_{0}\right) f_{b}(\Delta r)= \\
& Z_{e b}\left(r, \Omega_{0}\right) .
\end{aligned}
$$

Previous results have been obtained for a single homogeneous slab such that $L=0$. The expression of the backscattered radiance can be further generalised in a straightforward manner to a relationship valid for the any range bin of a layered atmosphere at distance $r$, that is:

$$
\begin{aligned}
& <P_{R}\left(r, \Omega_{0}\right)>\cong C \frac{Z_{a F O S}\left(r, \Omega_{o}\right)}{r^{2}}= \\
& C \frac{Z_{e b}\left(r, \Omega_{0}\right)}{r^{2}} L^{2}(r) \equiv C \frac{Z_{m}\left(r, \Omega_{0}\right)}{r^{2}},
\end{aligned}
$$

obtaining an equation formally similar to the classical radar equation (1), but with a measured reflectivity factor $Z_{m}$ including both the two-way path attenuation $L$ and the rangebin extinction factor $f_{b}$.

\section{Impact of range-bin extinction on rain radar observa- tions}

In this section, some numerical results of the theoretical analysis, illustrated above, will be shown. Examples are based on the use of statistical-empirical relationships, available for microwave and millimetre-wave radar observations of rainfall.

The relations among the equivalent reflectivity factor $Z_{e}$, the specific attenuation $k$ and the rain rate $R$ are well documented in literature. These relations are generally assumed to have a power-law form (e.g, Atlas et al., 1990):

$\left\{\begin{array}{l}Z_{e}=a R^{b} \\ k=c R^{d}\end{array}\right.$

where $a, b, c$ and $\mathrm{d}$ are regression coefficients. The latter are usually derived from the statistical analysis of in situ data, acquired by rain gauges, disdrometers and aircraft probes (e.g., Sauvageot, 1992; Bringi and Chandrasekar, 2001). An impressive large set of power-law relationships is available from literature - a variability which mainly depends (apart from observational effects) on the influence of raindrop size distributions related not only to the rain type and ambient temperature, but also raindrop terminal velocity and precipitation microphysical processes.

We have considered here various operational frequencies to evaluate the first-order scattering effects through the analysis of the range-bin extinction factor. As previously mentioned, ground- based applications are generally focused on the use of $\mathrm{C}, \mathrm{X}$ and $\mathrm{Ka}$ band, while airborne and spaceborne radars have been deployed at $\mathrm{Ku}$ and $\mathrm{Ka}$ band. For sake of completeness we show the numerical results for rainrate values up to $100 \mathrm{~mm} / \mathrm{h}$, even though the radar sensitivity will significantly decrease with the increase of frequency. For instance, a Ka-band spaceborne radar will be limited to about 

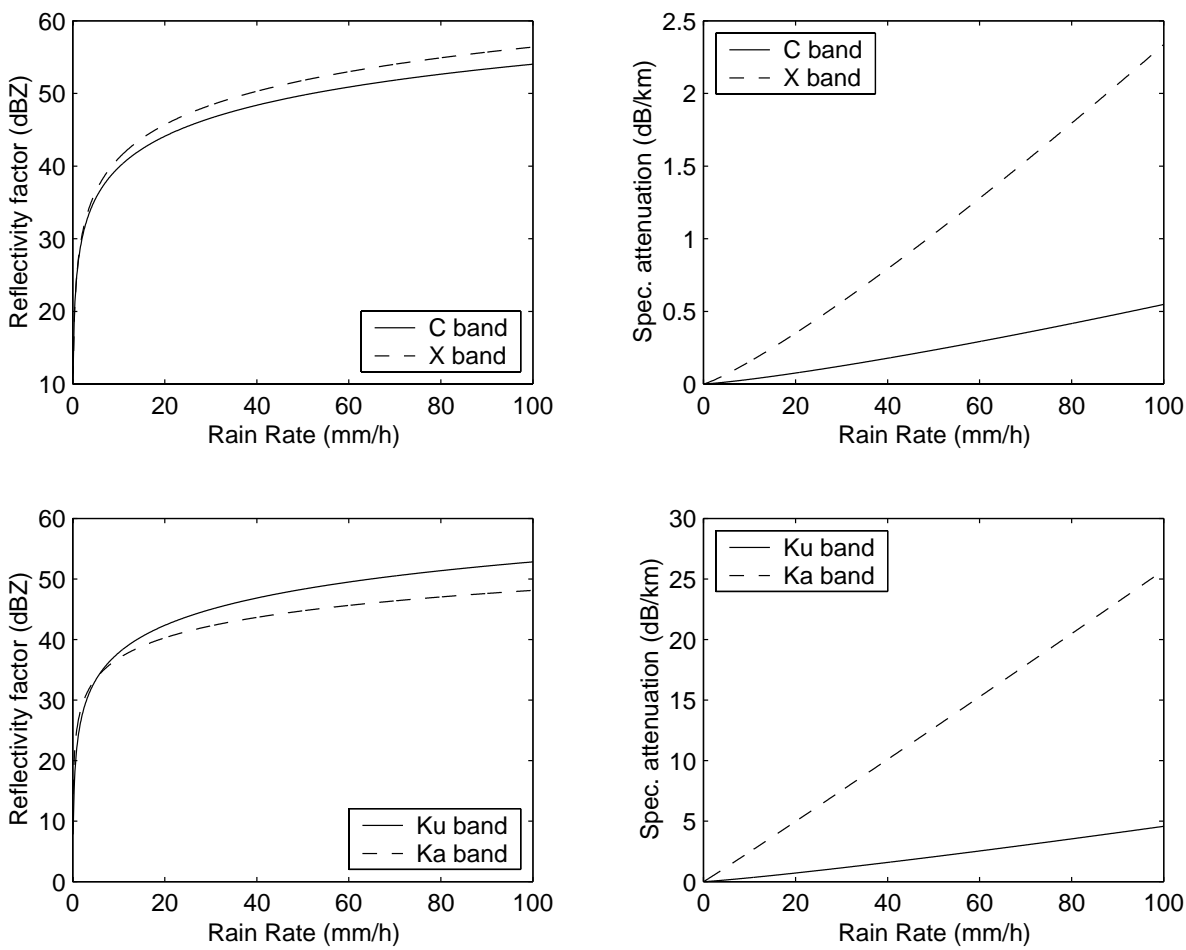

Fig. 1. Statistical power laws relating the equivalent reflectivity factor $Z_{e}$ and the specific attenuation $k$ to rain rate $R$, derived from Delrieu et al. (2000) at C and X band for orographic rainfall and from Haddad et al. (1997) at Ku and Ka band for tropical rainfall.

$10-20 \mathrm{~mm} / \mathrm{h}$ for acceptable signal-to-noise ratios (Meneghini et al., 1983).

\subsection{Numerical results}

From open literature, we have selected $Z_{e}-R$ and $k-R$ relations relative to orographic rainfall at $\mathrm{C}$ and $\mathrm{X}$ band, derived by Delrieu et al., 2000 (i.e., their Table 2 at $0^{\circ} \mathrm{C}$ for "Cévennes" model). At Ku and Ka band, we have resorted to tropical rainfall scenarios by using $Z_{e}-R$ and $k-R$ from Haddad et al., 1997 (their Tables IX-XII for D"=1.0). Figure 1 shows the behaviour of these power-law relations for the four frequency bands as a function of the rain rate $R$.

By definition, $f_{b}$ is dependent on the optical thickness (or total attenuation) $\Delta \tau_{r}$ of the range bin. Thus, for a given $R$, $k$ being determined by (28) (see Fig. 1), we need to specify the range bin resolution $\Delta r$. The latter, in accordance with common operational requirements, has been set to 125,250 , 500 and $1000 \mathrm{~m}$, respectively.

Figure 2 illustrates the behaviour of the range-bin extinction factor $f_{b}$ as a function of $R$ for the same frequency bands of Fig. 1, setting $\Delta r$ as a parameter. It should be noted that, in the case of negligible range bin extinction, $f_{b}=1$ (or $\left.f_{b}=0 \mathrm{~dB}\right)$.

As expected, for a given rain rate, the range-bin extinction factor $f_{b}$ is less than 1 (or less than $0 \mathrm{~dB}$ ) as the frequency and range resolution increase. While at $\mathrm{C}$ band, $f_{b}$ is always higher than $-1 \mathrm{~dB}$ up to $100 \mathrm{~mm} / \mathrm{h}$, at Ka band $f_{b}<-1 \mathrm{~dB}$ for $R>20 \mathrm{~mm} / \mathrm{h}$ and $\Delta r>250 \mathrm{~m}$. These results, thus, confirm that the effect of the range-bin extinction factor, depending on the frequency and range resolution, can be significant for intense rain and, at Ka band and above, even for moderate rain.

In order to show the impact of rainfall regime on the evaluation of the range-bin extinction factor, Fig. 3 shows the same as in Fig. 2, but for a $k-R$ relation typical of widespread rain and thunderstorm rain at $\mathrm{C}$ and $\mathrm{X}$ band, as derived by Delrieu et al., 2000 (i.e., their Table 2 at $0^{\circ} \mathrm{C}$ for "widespread" and "thunderstorm" models).

From the comparison of Fig. 3 with Fig. 2, it emerges that values of $f_{b}$ for widespread rain are less than those relative to orographic rainfall, as reasonable. On the contrary, for thunderstorm rain at $\mathrm{C}$ band $f_{b}$ can be less than $-0.7 \mathrm{~dB}$ at $100 \mathrm{~mm} / \mathrm{h}$ and $\Delta r=1000 \mathrm{~m}$, while at $\mathrm{X}$ band $f_{b}$ can be less than $-0.5 \mathrm{~dB}$ at $50 \mathrm{~mm} / \mathrm{h}$ and for a range resolution of $500 \mathrm{~m}$.

\subsection{Discussion}

The theoretical and numerical analysis, illustrated so far, has evidenced a possible impact of the range-bin extinction on radar observations of rainfall at attenuating wavelength. Indeed, as shown elsewhere, graupel and hail back scattering in convective systems can cause a significant albedo thus contributing to increase of the specific attenuation within range 

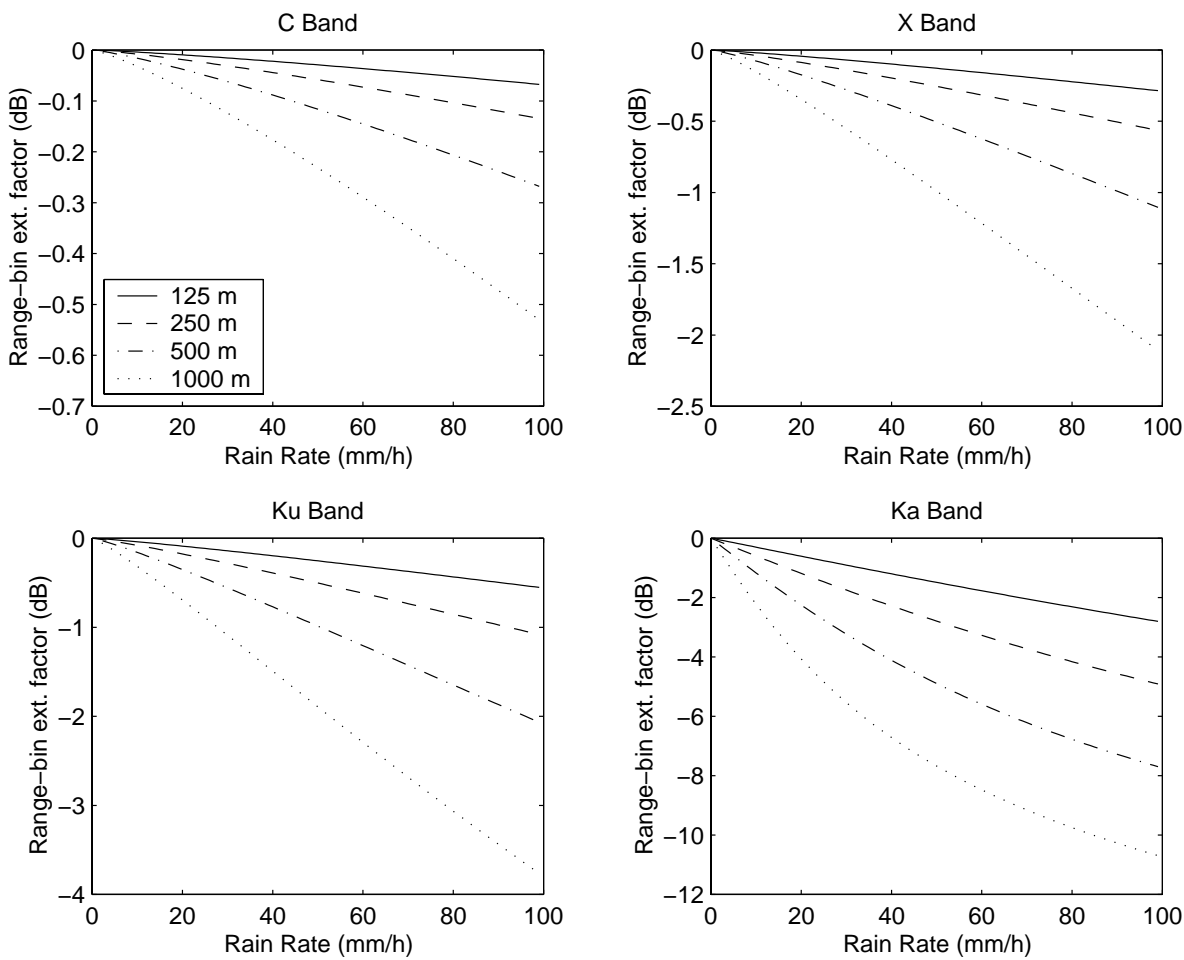

Fig. 2. Range-bin extinction factor $f_{b}$ as a function of the rain rate $R$, derived using the relationships plotted in Fig. 1, with the range-bin resolution $\Delta r$ as a parameter (ranging from $125 \mathrm{~m}$ to $1000 \mathrm{~m}$ ).
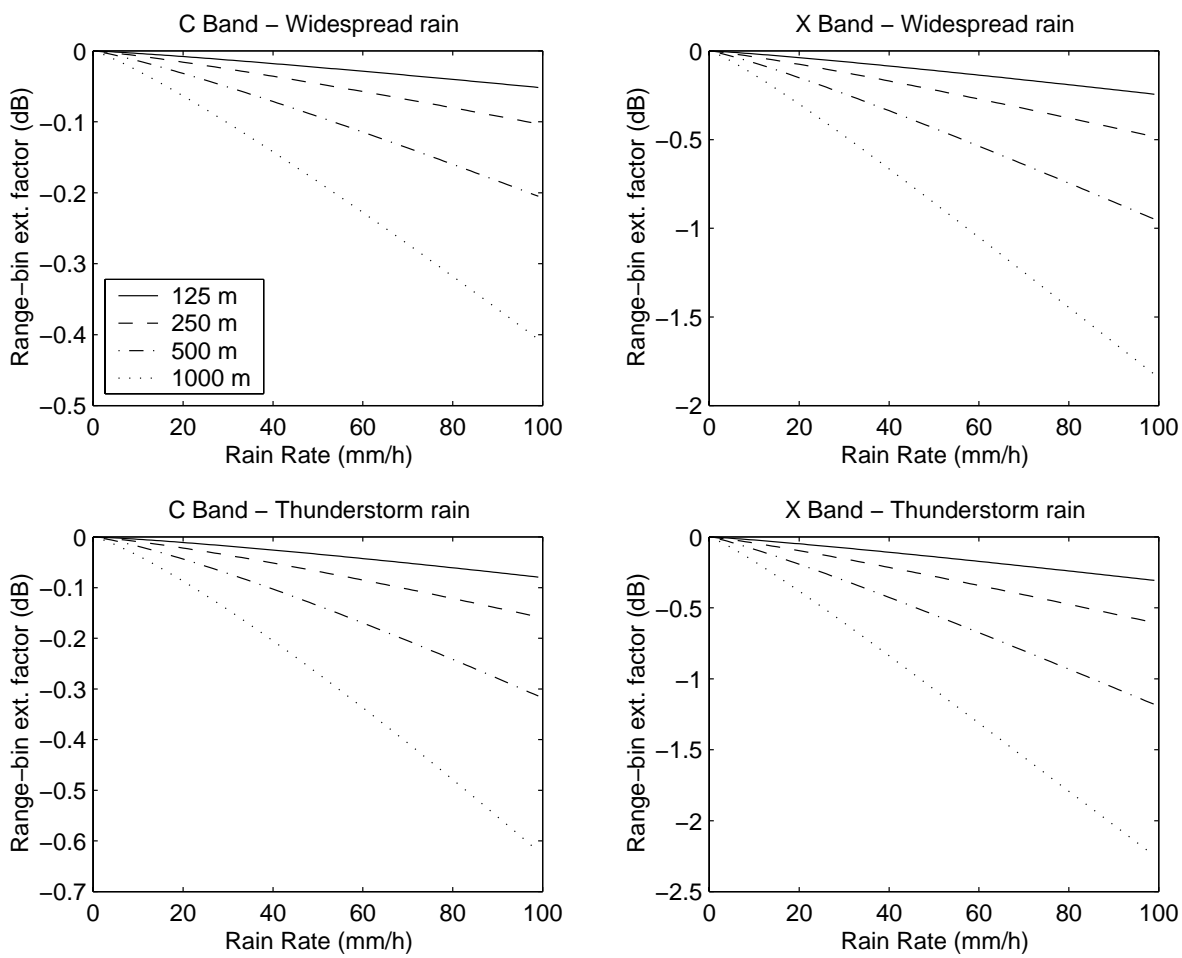

Fig. 3. Same as in Fig. 2, but for $k-R$ relations typical of widespread rain and thunderstorm rain at $\mathrm{C}$ and $\mathrm{X}$ band, as derived by Delrieu et al., 2000. 
bins where rain is not present such as above the freezing level (Marzano et al., 2003). This is especially important, of course, for airborne and spaceborne rain radars.

Secondly, if the range-bin extinction factor is less than 1 for various reasons (i.e., large bins and/or high attenuation), it emerges that the retrieval algorithms to estimate rainfall from radar observations at attenuating wavelength can be affected as well. In fact, by assuming at each range bin the validity of (28), from (27) and (4) the general form of the integral equation to be inverted to retrieve the equivalent reflectivity factor $Z_{e}$ from a measured one $Z_{m}(r)$ at a range $r$ is:

$$
\begin{aligned}
& Z_{m}\left(r, \Omega_{0}\right)= \\
& Z_{e}\left(r, \Omega_{0}\right) f_{b}(r) e^{-2 \int_{0}^{r} \alpha\left[Z_{e}\left(r, \Omega_{0}\right)\right]^{\beta} d r^{\prime}},
\end{aligned}
$$

where, eliminating $R$ from (28), it holds:

$k=\alpha Z_{e}^{\beta}$,

being $\alpha$ and $\beta$ are related to $a, b, c$ and $d$ coefficients of (28).

From (29) it is clear that, only if $f_{b}=1$, well-known physical inversion techniques can be applied to retrieve $Z_{e}$, and by (28), rainfall rate $R$ (e.g., Meneghini et al., 1983; Aydin et al., 1989; Testud et al., 2000). In all other cases, the role of the range-bin extinction should be considered at least as a multiplicative noise which inevitably deteriorates the final accuracy of the rain estimate (Marzano et al., 2003). When assuming (28) as in Fig. 1 and $\Delta r=250 \mathrm{~m}$, numerical tests (not shown here for brevity) indicate that this error may range from $15 \%$ at $\mathrm{X}$ band at $100 \mathrm{~mm} / \mathrm{h}$ to $15 \%$ at $\mathrm{Ku}$ at $50 \mathrm{~mm} / \mathrm{h}$ and $20 \%$ at Ka band at $10 \mathrm{~mm} / \mathrm{h}$. Note that if $r$ refers to the range bin centre (and not to its edge), these potential errors can be empirically reduced.

The final consideration is related to the impact of rangebin extinction when performing a spatial averaging of radar reflectivity data in strong attenuation conditions. In order to illustrate this point, let us suppose for simplicity to operate at $X$ band observing a uniform rain slab with $50 \mathrm{~mm} / \mathrm{h}$, characterized by a constant $Z_{e 0}$. From Fig. 2, we see that, for $\Delta r=125 \mathrm{~m}, f_{b}=0.98$ (i.e., about $-0.1 \mathrm{~dB}$ ), while for $\Delta r=1 \mathrm{~km} f_{b}$ is about 0.79 (i.e., about $-1.0 \mathrm{~dB}$ ). This means that, if the measured reflectivity is averaged using $N$ range bins, for its spatial average it holds:

$\bar{Z}_{e b}=Z_{e 0} f_{b}(r ; N \Delta r) \neq$

$$
Z_{e 0} \frac{\sum_{i=1}^{N} f_{b}^{(i)}(r ; \Delta r)}{N} \cong Z_{e 0}
$$

The difference between the left and right-hand side of inequality (31) is, in the above mentioned conditions, about $-1 \mathrm{~dB}$.

Even though $-1 \mathrm{~dB}$ seems to be a negligible effect, it is worth noting that, due to the power-law relation between $Z_{e}$ and $R$ in (28), it can correspond to an underestimation of about $7 \mathrm{~mm} / \mathrm{h}$ over $50 \mathrm{~mm} / \mathrm{h}$, that is an error of $15 \%$. Since it is operationally fairly common to provide products at a degraded range resolution, the last inequality should be kept in mind when processing radar data in attenuating media.

\section{Summary and conclusions}

The meteorological radar equation in its classical form takes into account two-way path attenuation from each range bin to the radar antenna. Indeed, for strong attenuating media, such as intense rainfall at microwaves, hydrometeor extinction within each range bin can be significant as well. A modified radar equation has been here derived from the integral radar equation to include such a range-bin extinction effect. It has been shown that, only in the case of low-to-moderate attenuating media, the derived range-bin extinction factor is, by definition, closed to one so that the classical radar equation can be used.

These theoretical results have also been obtained by using a microwave radiative transfer approach, taking into account the multiple scattering effects. It has been shown that a new definition of the radar reflectivity, in terms of backscattered specific intensity, yields a generalised radar equation. The latter, under the assumption of first-order scattering, reduces to the modified radar equation, previously obtained. These theoretical results have supported the conclusion that radar analysis in strong attenuating media should include, in general, first-order scattering effects. On the other hand, they prove that the classical radar equation takes into account firstorder backscattering in the limit of negligible range-bin extinction.

Numerical simulations have been performed by using statistical power laws relating the radar reflectivity to rain rate and specific attenuation, derived from experimental data and available in the open literature. Results confirm that the effect of the range-bin extinction factor, depending on the considered frequency and range resolution, can be relevant at $\mathrm{X}$ band for intense rain, while at Ka band and above can be appreciable even for moderate rain. These errors, even though small in some observational circumstances, can have a strong impact in determining the accuracy of radar retrieval algorithms, aimed at correcting two-way rainfall path attenuation, and on spatial averaging procedures applied to radar reflectivity data in relevant attenuation conditions.

Acknowledgements. This work has been partially supported by the Italian Space Agency (ASI), the Italian National Research Council (CNR) through GNDCI project and the Italian Ministry of Education, University and Research (MIUR). 


\section{References}

Atlas, D. (Ed): Radar in Meteorology, American Meteorological Society, Boston, 1990.

Aydin, K., Zhao, Y., and Seliga, T. A.: Rain-Induced Attenuation Effects on C-Band Dual-Polarization Meteorological Radars, IEEE Trans. on Geosci. Remote Sens., 27, 1, 57-66, 1989.

Bogush, A. J.: Radar and the atmosphere, Artech House, Norwood (MA), 1989.

Bringi, V. N. and Chandrasekar, V.: Polarimetric Doppler Weather Radar: principles and applications, Cambridge University Press, Cambridge (MA), 2001.

Delrieu, G., Andrieu, H., and Creutin,J. D.: Quantification of PathIntegrated Attenuation for X- and C-Band Weather Radar Systems Operating in Mediterranean Heavy Rainfall, J. Appl. Meteor., 39, 840-850, 2000.

de Wolf, D. A., Russchenberg, H. W. J., and Ligthart, L. P.: Radar reflection from clouds: Gigahertz backscatter cross sections and Doppler spectra, IEEE Trans. Antennas Propagat., 48, 254-259, 2000.

Haddad, Z. S., Short, D. A., Durden, S. L., Im, E., Hensley, S., Grable, M. B., and Black, R. A.: A new raindrop parametrization of the raindrop size distribution, IEEE Trans. Geosci. Remote Sens., 35, 532-539, 1997.

Ishimaru, A.: Wave propagation and scattering in random media, Vol. 1 e 2, Academic Press, New York (NY), 1978.

Marzano, F. S., Mugnai, A., Panegrossi, G., Pierdicca, N., Smith, E. A., and Turk, J.: Bayesian estimation of precipitating cloud parameters from combined measurements of spaceborne microwave radiometer and radar, IEEE Trans. Geosci. Remote Sens., 37, 596-613, 1999.
Marzano F. S., Fionda, E., and Ciotti, P.: Simulation of radiometric and attenuation measurements along earth-satellite links in the 10- to $50-\mathrm{GHz}$ band through horizontally-finite convective raincells", Radio Sci., 34, 841-858, 1999.

Marzano, F. S., Roberti, L., and Mugnai, A.: Impact of Rainfall Incoherent Backscattering Upon Radar Echoes Above $10 \mathrm{GHz}$, Phys.Chem. Earth (B), 25, No. 10-12, 943-948, 2000.

Marzano, F. S. and Ferrauto, G.: Investigation on the meteorological radar equation at attenuating wavelength, Proc. ERAD'02, Delft (NL), 18-21 Nov. 2002.

Marzano F. S., Roberti, L., Di Michele, S., Tassa, A., and Mugnai, A.: Modeling of apparent radar reflectivity due to convective clouds at attenuating wavelengths, Radio Sci., 38, 10.1029/2002RS002613, 2003.

Meneghini, R.: Rain-rate Estimates for an Attenuating Radar, Radio Science, 13, 459-470, 1978.

Meneghini, R., Eckerman, J., and Atlas, D.: Determination of Rain Rate from a Space-borne Radar Using Measurement of Total Attenuation, IEEE Trans. on Geosci. Remote Sens., 21, 34-43, 1983.

Sauvageot, H.: Radar Meteorology, Artech House, Norwood (MA), 1992.

Serrar, S., Delrieu, G., Creutin, J. D., and Uijlenhoet, R.: Mountain reference technique: Use of mountain returns to calibrate weather radars operating at attenuating wavelengths, J. of Geophys. Research, 105, No. D2, 2281-2290, 2000.

Testud, J., Le Bouar, E., Obligis, E., and Ali-Mehenni, M.: The Rain Profiling Algorithm Applied to Polarimetric Weather Radar, J. Atmospheric and Oceanic Techn., 17, 332-356, 2000.

Tsang, L., Kong, J. A., and Shin, R. T.: Theory of microwave remote sensing, J. Wiley \& Sons, New York (NY), 1985. 